\title{
Enzymatic Template Polymerization of Phenol in the Presence of Water-soluble Polymers in an Aqueous Medium
}

\author{
Young-Jin KIM, Hiroshi UyAMA, ${ }^{\dagger}$ and Shiro KOBAYASHI ${ }^{\dagger \dagger}$ \\ Department of Materials Chemistry, Graduate School of Engineering, Kyoto University, Kyoto 615-8510, Japan
}

(Received August 9, 2004; Accepted September 28, 2004; Published December 15, 2004)

\begin{abstract}
The enzymatic polymerization of phenol was investigated by using water-soluble polymers (WSPs) as template in an aqueous medium. The addition of PEG, one of WSPs, produced a miscible complex of polyphenol and PEG as precipitates in high yields. CD measurement verified the formation of a phenol-PEG complex by hydrogen bonding interaction. The presence of PEG in an aqueous medium greatly improved the regioselectivity of the polymerization, yielding a polyphenol with the phenylene unit content higher than $90 \%$. The amount of PEG strongly affected the polyphenol yield. The unit molar ratio of polyphenol and PEG was $c a$. 1:1. The FT IR, DSC, and XRD analyses exhibited the formation of the miscible complex of polyphenol and PEG by hydrogen bonding interaction.

[DOI 10.1295/polymj.36.992]

KEY WORDS Enzymatic Polymerization / Poly(ethylene glycol) / Polyphenol / Regioselectivity / Template Polymerization / Water-soluble Polymer /
\end{abstract}

Polymerization of a monomer in the presence of a preformed macromolecule is usually defined as template polymerization. ${ }^{1}$ The presence of the template can influence the polymerization rate of the monomer with respect to the blank polymerization and also properties of the final product such as molecular weight and stereoregularity. Template polymerization leads to formation of polymer-polymer complexes stabilized via noncovalent binding forces like electrostatic interactions, hydrogen bonds, hydrophobic interactions, and stereocomplexation. ${ }^{2}$ It is accepted that template polymerization proceeds according to two different mechanisms, zip and pick-up mechanisms. ${ }^{1}$ Usually, when the monomers are connected with the template by strong forces such as hydrogen and electrostatic bonds, the polymerization starts through a zip mechanism. ${ }^{1,2}$ However, strict control of the free radical polymerization by using the template has not successfully been achieved even in the case of a linear template polymer with a narrow molecular weight distribution. This is probably because the free radical polymerization is randomly initiated in the template.

Recently, enzymatic syntheses of phenolic polymers have received much attention as an alternative process for preparing conventional phenolic resins without the use of toxic formaldehyde, a monomer for production of conventional phenolic resins (phenol-formaldehyde resins). ${ }^{3}$ So far, a new class of useful and high-performance polyphenols have been prepared by utilizing characteristic catalysis of enzymes, most of which can not be obtained by conventional chemical methods. ${ }^{4}$ In the enzymatic oxidative poly- merization of phenol derivatives, however, control of the coupling selectivity (regioselectivity) is often very difficult, since the reaction proceeds via coupling of free radical intermediates. From phenol, the simplest and most important phenolic compound in industrial fields, a soluble polyphenol consisting of a mixture of phenylene and oxyphenylene units was synthesized in a mixture of methanol and phosphate buffer (Scheme 1). ${ }^{5}$ The regioselectivity depended on the mixed ratio of methanol and the buffer to form the polyphenol in the range of the phenylene content from 40 to $70 \%$. However, the regioselective synthesis of poly(phenylene) or poly(oxyphenylene) from phenol has not been achieved yet.

For the development of an environmentally benign process of polymer production, the use of organic solvents is not preferred. However, the enzymatic polymerization of phenol scarcely proceeds in a buffer; the monomer was quantitatively converted to the polyphenol in a mixture of water-miscible organic solvent and buffer. ${ }^{5}$ The polymerization of phenol and $m$-substituted phenols in the presence of cyclodextrin derivatives in a buffer was reported. ${ }^{6}$ The addition of cyclodextrin induced the polymerization in the aqueous medium to produce soluble polyphenols in high

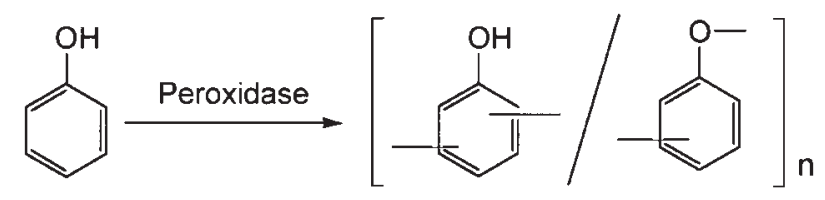

Scheme 1.

\footnotetext{
${ }^{\dagger}$ Present Address: Department of Materials Chemistry, Graduate School of Engineering, Osaka University, Suita, 565-0871, Japan

${ }^{\dagger}$ To whom correspondence should be addressed (Tel: +81-75-383-2459, Fax: +81-75-383-2461, E-mail: kobayasi@mat.polym.kyoto-u.ac.jp).
} 


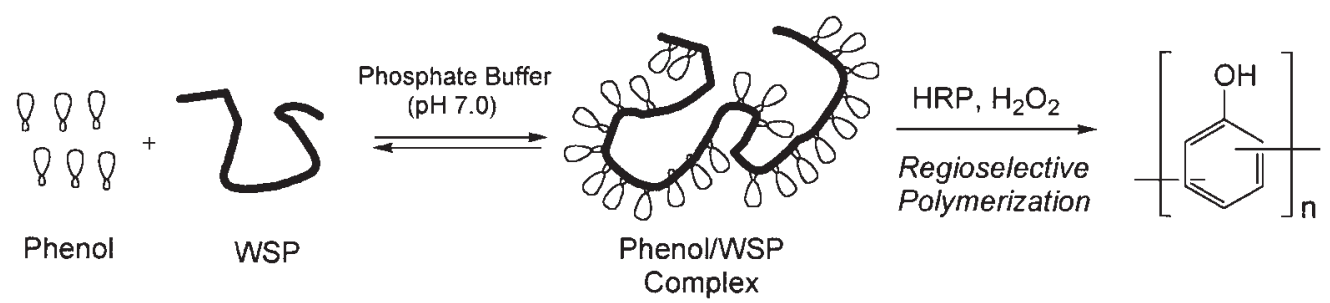

Scheme 2.

yields. However, a relatively much amount of cyclodextrin was required for the efficient production of the polyphenol. In the product polymer, a small amount of cyclodextrin was contained.

Recently, poly(sodium 4-styrene sulfonate) $(\mathrm{PSS})^{7}$ and poly(ethylene glycol) $(\mathrm{PEG})^{8}$ were reported to act as template in the enzymatic polymerization of phenol in water, yielding a complex of the polyphenol and the template polymer. When PEG was used as template, the regioselectivity greatly improved to give the polymer mainly consisting of the phenylene unit. ${ }^{8 b}$ This study deals with the enzymatic template polymerization of phenol using water-soluble polymers (WSPs) in a buffer and properties of the product polymers (Scheme 2).

\section{EXPERIMENTAL}

\section{Materials}

Horseradish peroxidase (HRP), phenol and PEG were purchased from Wako Pure Chemical Industries, Ltd. and used without further purification. $\operatorname{Poly}(N-$ vinylpyrrolidone) (PVP) and poly(vinyl methyl ether) (PVME) were obtained from Tokyo Kasei Kogyo Co., Ltd. Poly(2-ethyl-2-oxazoline) (PEOZ) was purchased from Aldrich. Poly(2-methyl-2-oxazoline) (PMOZ) was prepared according to the literature. ${ }^{9}$ Poly(vinyl alcohol) (PVA, degree of saponification: $88 \%$ ) was kindly donated by Kuraray Co., Ltd. Other reagents and solvents were commercially available and were used as received.

\section{Synthesis of Polyphenol}

A typical run was as follows (entry 1 in Table I). Phenol $(0.47 \mathrm{~g}, 5.0 \mathrm{mmol})$ and PEG $(0.47 \mathrm{~g}, 10.7$ mmol of monomer unit) were dissolved in $0.1 \mathrm{M}$ phosphate buffer $(\mathrm{pH} 7.0)(20 \mathrm{~mL})$. Then, the solution of HRP (2.0 mg, 440 units) in $0.1 \mathrm{M}$ phosphate buffer $(5 \mathrm{~mL})$ was added. To this solution, $3.4 \mathrm{~mL}$ of $5 \%$ hydrogen peroxide $(5.6 \mathrm{mmol})$ was added dropwise for $2 \mathrm{~h}$. The mixture was stirred at room temperature under air. After $1 \mathrm{~h}$, the precipitated polymeric materials were collected by centrifugation and washed with water repeatedly, followed by drying in vасио to give the polymer.
${ }^{1} \mathrm{H}$ NMR (DMSO- $\left.d_{6}\right): \delta 3.3-3.6\left(\mathrm{~m}, \mathrm{CH}_{2} \mathrm{CH}_{2} \mathrm{O}\right)$, 6.6-7.4 (br, Ar), 9.2-9.6 (br, ArOH).

${ }^{13} \mathrm{C}$ NMR (DMSO- $\left.d_{6}\right): \delta 70$ (C-C-O), 110-120 (Ar), 127-135 (Ar), 155-165 (Ar).

FT IR (KBr): 3289 ( $v$ O-H), 1587, 1489, 1448 ( $v$ $\mathrm{C}=\mathrm{C}$ of Ar$), 1206(v \mathrm{C}(\mathrm{Ar})-\mathrm{O}-\mathrm{H}), 1098 \mathrm{~cm}^{-1}(v$ $\mathrm{CH}_{2}-\mathrm{O}-\mathrm{CH}_{2}$ ).

\section{Measurements}

Size exclusion chromatographic (SEC) analysis was carried out by using a Tosoh GPC-8020 apparatus equipped with refractive index (RI) and UV detectors under the following conditions: TSKgel $\alpha-3000$ and $\alpha$-M columns and DMF containing $0.10 \mathrm{M} \mathrm{LiCl}$ eluent at a flow rate of $1.00 \mathrm{~mL} / \mathrm{min}$ at $60^{\circ} \mathrm{C}$. The calibration curves were obtained using polystyrene as standard. NMR spectra were recorded on a Bruker DPX400 spectrometer. FT IR measurements were carried out with a PerkinElmer Spectrum One. DSC analysis was performed using a Seiko SSC5200-DSC220 apparatus at a heating rate of $10^{\circ} \mathrm{C} / \mathrm{min}$ in a nitrogen flow rate of $30 \mathrm{~mL} / \mathrm{min}$. Circular dichroism (CD) spectra were recorded on a Jasco J-820 spectropolarimeter at $25^{\circ} \mathrm{C}$ in a nitrogen flow rate of $5 \mathrm{~mL} / \mathrm{min}$. X-ray diffraction (XRD) measurements were carried out with a Rigaku X-ray diffractometer RINT 2500 with $\mathrm{Cu} \mathrm{K} \alpha$ radiation at $50 \mathrm{kV} / 300 \mathrm{~mA}$. The diffractograms were scanned in a $2 \theta$ range of $10-40^{\circ}$ at a rate of $2^{\circ} / \mathrm{min}$.

\section{RESULTS AND DISCUSSION}

Enzymatic Polymerization of Phenol in the Presence of Water-soluble Polymers

At first, we carried out the HRP-catalyzed polymerization of phenol in a phosphate buffer $(\mathrm{pH} 7)$ in the presence of various water-soluble polymers (WSPs) such as PEG, poly(vinyl methyl ether) (PVME), poly(vinyl alcohol) (PVA), poly( $N$-vinylpyrrolidone) (PVP), poly(2-methyl-2-oxazoline) (PMOZ), poly(2ethyl-2-oxazoline) (PEOZ), and poly(sodium 4-styrene sulfonate) (PSS) at room temperature under air (Table I). The polymerization started by the addition of hydrogen peroxide as an oxidizing agent. In most cases, powdery precipitates were formed during the 
Table I. Polymerization of phenol with $\mathrm{WSP}^{\mathrm{a}}$

\begin{tabular}{clccccc}
\hline Entry & WSP & $\begin{array}{c}\text { Molecular weight } \\
\text { of WSP }\left(\times 10^{3}\right)\end{array}$ & $\begin{array}{c}\text { Yield } \\
(\%)^{\mathrm{c}}\end{array}$ & $M_{\mathrm{n}}{ }^{\mathrm{d}}$ & $\begin{array}{c}\text { Composition ratio } \\
\left(\text { Polyphenol:WSP) }^{\mathrm{e}}\right.\end{array}$ & ${\mathrm{Ph} / \mathrm{Ox}^{\mathrm{f}}}^{\mathrm{b}}$ \\
\hline 1 & PEG & $0.4(9)$ & 85 & 1600 & $1: 0.59$ & $89 / 11$ \\
2 & PEG & $2(45)$ & 94 & 2300 & $1: 1.00$ & $88 / 12$ \\
3 & PVME & $46(790)$ & $-{ }_{\mathrm{g}}$ & - & - & - \\
4 & PVA & $91(2000)$ & $-{ }^{\mathrm{h}}$ & - & - & - \\
5 & PVP & $40(360)$ & $-\mathrm{g}$ & - & $1: 0.54$ & $74 / 26$ \\
6 & PMOZ & $0.9(9)$ & 78 & 1300 & $1: 1.02$ & $72 / 28$ \\
7 & PEOZ & $5(50)$ & 91 & 1600 & $1: 0.03$ & $34 / 76$ \\
8 & PSS & $70(340)$ & 70 & 2700 & - & - \\
9 & - & - & $\leq 3$ & - & - & - \\
\hline
\end{tabular}

${ }^{\text {a} P o l y m e r i z a t i o n ~ o f ~ p h e n o l ~}(0.47 \mathrm{~g}, 5.0 \mathrm{mmol})$ using HRP catalyst $(2.0 \mathrm{mg})$ in the presence of WSP $(0.47 \mathrm{~g})$ in $0.1 \mathrm{M}$ phosphate buffer ( $\mathrm{pH} 7.0$ ) at room temperature under air. ${ }^{\mathrm{b}}$ In parentheses, degree of polymerization of WSP. ${ }^{c}$ Yield of polyphenol based on the phenol part in the complex product, determined by ${ }^{1} \mathrm{H}$ NMR spectroscopy using acetone as the internal standard. ${ }^{\mathrm{d}}$ Determined by SEC using DMF as eluent with polystyrene standards. ${ }^{e}$ Molar ratio of monomer unit between polyphenol and WSP determined by ${ }^{1} \mathrm{H}$ NMR spectroscopy using acetone as the internal standard. ${ }^{\mathrm{f}}$ Unit ratio of phenylene/oxyphenylene $(\mathrm{Ph} / \mathrm{Ox})$ determined by titration of hydroxyl group. ${ }^{\mathrm{g}}$ Gelation took place by mixing of phenol and the template polymer. ${ }^{\mathrm{h}}$ Yield of polyphenol could not be determined because of the insolubility of the product in any solvents.

polymerization, which were collected by centrifugation after the polymerization. The unit ratio of phenylene/oxyphenylene $(\mathrm{Ph} / \mathrm{Ox})$ was determined by titration of the phenolic hydroxy group in the polymer. ${ }^{10}$

In the absence of WSP (entry 9), the polymer yield was very low. On the other hand, the polymer was obtained in high yields by the addition of PEG, PMOZ, PEOZ, and PSS. ${ }^{1} \mathrm{H}$ NMR analysis showed that the product was a complex of the phenolic polymer and a WSP. Among them, PEG enormously improved the regioselectivity to give the polymer mainly consisting of phenylene unit. The regioselectivity of the polymers obtained by using poly(2-oxazoline) derivatives as template was somewhat lower than that by PEG. In contrast, the ratio of the oxyphenylene unit was larger than that of the phenylene unit when PPS was used as template. These data suggest that the structure of the template polymer strongly affected the regioselectivity.

In the presence of PVA, the polymeric precipitates were also formed; however, the product was not soluble in any solvents. In the case of PVP or PVME, gelation immediately took place by mixing of phenol and the template polymer, probably due to the strong interaction between the phenol monomer and these polymers. These data clearly show that PEG was the most effective template for the regioselective synthesis of soluble polyphenols. Thus, we used PEG for the following experiment.

\section{Role of PEG}

On the basis of the above results, we focused on the role of PEG in the oxidative polymerization of phenol in an aqueous medium. The change of CD spectra of phenol was observed in the presence and absence of PEG $\left(M_{\mathrm{n}}=2 \times 10^{4}\right)$ in phosphate buffer ( $\mathrm{pH} 7.0$ ). The CD spectrum of phenol exhibited two negative bands at about 225 and $270 \mathrm{~nm}$. By adding PEG to the buffer containing phenol, the molar ellipticity of the negative CD band at $270 \mathrm{~nm}$ decreased. A similar behavior was not observed in the PEG solution of different concentration without phenol. These data suggest the formation of a phenol-PEG complex and the overall lowering of the $\pi \rightarrow \pi^{*}$ energy transition (Figure 1). ${ }^{11}$ This energy transition is most likely lowered by non-bonded electrons of the ether groups of PEG withdrawing electrons from the molecular orbitals of phenol by the hydrogen bonding interaction. The molar ellipticity of the CD band at $270 \mathrm{~nm}$ de-

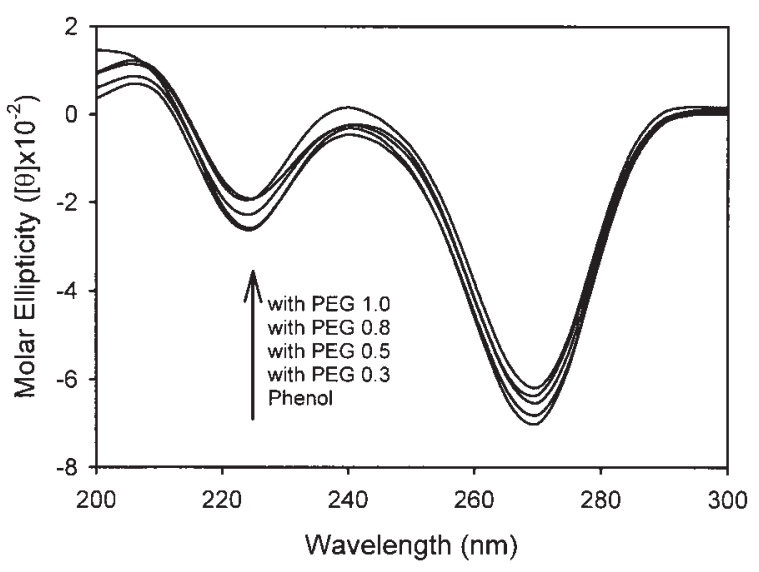

Figure 1. CD spectra of phenol and phenol-PEG $\left(M_{\mathrm{n}}=\right.$ $2 \times 10^{4}$ ) complexes with different weight ratio of PEG to phenol amount in phosphate buffer ( $\mathrm{pH} 7.0)$. The numbers of legend in the figure are weight ratios of PEG to phenol. 
Enzymatic Template Polymerization of Phenol

Table II. Polymerization of phenol with different PEG amount and molecular weight ${ }^{\mathrm{a}}$

\begin{tabular}{rcccccc}
\hline Entry & $\begin{array}{c}\text { Molecular } \\
\text { weight of PEG } \\
\left(\times 10^{3}\right)\end{array}$ & $\begin{array}{c}\text { PEG } \\
(\mathrm{g})^{\mathrm{b}}\end{array}$ & $\begin{array}{c}\text { Yield } \\
(\%)^{\mathrm{c}}\end{array}$ & $M_{\mathrm{n}}{ }^{\mathrm{d}}$ & $\begin{array}{c}\text { Composition ratio } \\
(\text { Polyphenol:PEG })^{\mathrm{e}}\end{array}$ & ${\mathrm{Ph} / \mathrm{Ox}{ }^{\mathrm{f}}}$ \\
\hline 1 & 2 & $0.1(0.45)$ & 44 & 2800 & $1: 1.02$ & $93 / 7$ \\
2 & 2 & $0.2(0.91)$ & 83 & 2300 & $1: 1.02$ & $95 / 5$ \\
3 & 20 & $0.1(0.45)$ & 23 & 3700 & $1: 1.04$ & $85 / 15$ \\
4 & 20 & $0.2(0.91)$ & 40 & 3500 & $1: 1.02$ & $83 / 17$ \\
5 & 20 & $0.3(1.4)$ & 59 & 3700 & $1: 1.06$ & $82 / 18$ \\
6 & 20 & $0.4(1.8)$ & 96 & 3800 & $1: 1.03$ & $84 / 16$ \\
7 & 20 & $0.5(2.3)$ & 93 & 3700 & $1: 1.05$ & $82 / 18$ \\
8 & 20 & $0.7(3.6)$ & 93 & 3700 & $1: 1.00$ & $80 / 20$ \\
9 & 0.2 & $0.47(2.2)$ & $\leq 3$ & - & - & $-1: 0.53$ \\
10 & 0.3 & $0.47(2.2)$ & 27 & 1600 & $1: 0.59$ & $82 / 8$ \\
11 & 0.4 & $0.47(2.2)$ & 85 & 1600 & $1: 0.80$ & $88 / 11$ \\
12 & 1 & $0.47(2.2)$ & 94 & 2000 & $1: 1.00$ & $88 / 12$ \\
13 & 2 & $0.47(2.2)$ & 94 & 2300 & $1: 1.03$ & $88 / 12$ \\
14 & 6 & $0.47(2.2)$ & 92 & 2200 & $1: 1.05$ & $82 / 18$ \\
15 & 20 & $0.47(2.2)$ & 93 & 3700 & $1: 1.07$ & $80 / 20$ \\
16 & 100 & $0.47(2.2)$ & 53 & 3700 & $1: 1.09$ & $77 / 23$ \\
17 & 200 & $0.47(2.2)$ & 40 & 3800 & - & - \\
18 & 300 & $0.47(2.2)$ & $\leq 2$ & - & & - \\
\hline
\end{tabular}

aPolymerization of phenol $(0.47 \mathrm{~g}, 5.0 \mathrm{mmol})$ using HRP catalyst $(2.0 \mathrm{mg})$ in the presence of PEG in $0.1 \mathrm{M}$ phosphate buffer ( $\mathrm{pH} 7.0)$ at room temperature under air. ${ }^{\mathrm{b}}$ In parentheses, molar ratio of monomer unit of PEG for phenol. ${ }^{c}$ Yield of polyphenol based on the phenol part in the complex product, determined by ${ }^{1} \mathrm{H}$ NMR spectroscopy using acetone as the internal standard. ${ }^{\mathrm{d}}$ Determined by SEC using DMF as eluent with polystyrene standards. ${ }^{\mathrm{e}}$ Molar ratio of monomer unit between polyphenol and PEG determined by ${ }^{1} \mathrm{H}$ NMR spectroscopy using acetone as the internal standard. ${ }^{\mathrm{f}}$ Unit ratio of phenylene/oxyphenylene $(\mathrm{Ph} / \mathrm{Ox})$ determined by titration of hydroxyl group.

creased with increasing the weight ratio of PEG to phenol, suggesting the hydrogen bonding interaction of PEG with the hydroxyl group of phenol through a zip mechanism. ${ }^{1,2}$ The formation of the phenol-PEG complex was previously confirmed by UV spectrometer by us; change of the specific peak intensity around $270 \mathrm{~nm}$ was observed by the addition of PEG to the buffer containing phenol. ${ }^{8 b}$

\section{Template Polymerization of Phenol in the Presence of PEG}

Polymerization results with using PEGs of different molecular weight are summarized in Table II. The PEG amount greatly affected the reaction behaviors. Figure 2 showed the effect of PEG $\left(M_{\mathrm{n}}=2 \times 10^{4}\right)$ amount on the yield and regioselectivity of the polymer. The polymer yield was low in the presence of PEG with amount less than $0.3 \mathrm{~g}$ for $0.47 \mathrm{~g}$ of the phenol monomer. When using more than $0.4 \mathrm{~g}$ of PEG, on the other hand, the polymer was obtained almost quantitatively. The high yield in using an excess of PEG in the molar basis is probably because the phenol-PEG complex is formed efficiently. In all cases, the unit molar ratio of polyphenol and PEG was $c a .1: 1$

In addition, the molecular weight of PEG affected

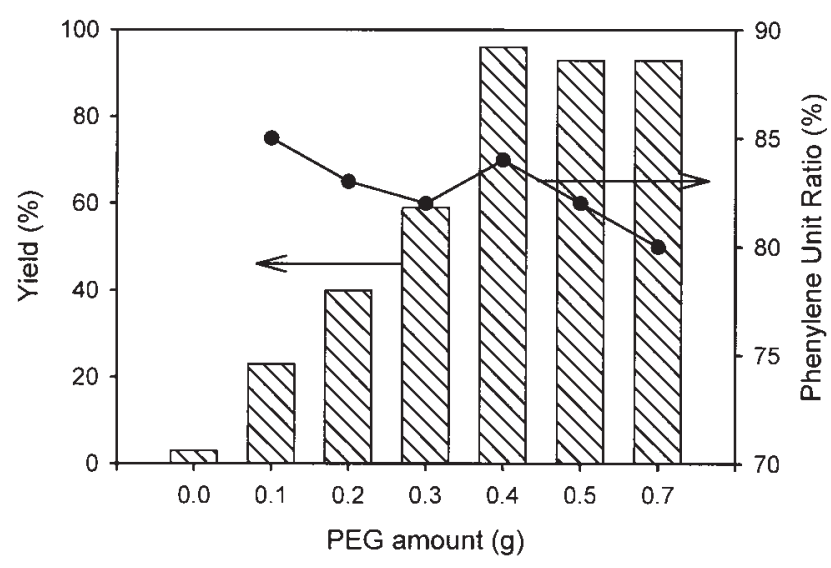

Figure 2. Effect of PEG $\left(M_{\mathrm{n}}=2 \times 10^{4}\right)$ amount on polymer yield and structure.

the yield and regioselectivity of the polymer (entries 9-18). In the range of the PEG molecular weight from $4 \times 10^{2}$ to $2 \times 10^{4}$, the yield was very high. On the other hand, the yield decreased enormously in the presence of PEG with molecular weight below $3 \times$ $10^{2}$ and above $1 \times 10^{5}$. The low yield in using lowmolecular-weight PEG is probably because the phenol-PEG complex is not formed adequately. The gelation and/or large increase of the solution viscosity were observed by the addition of PEG of high-molec- 


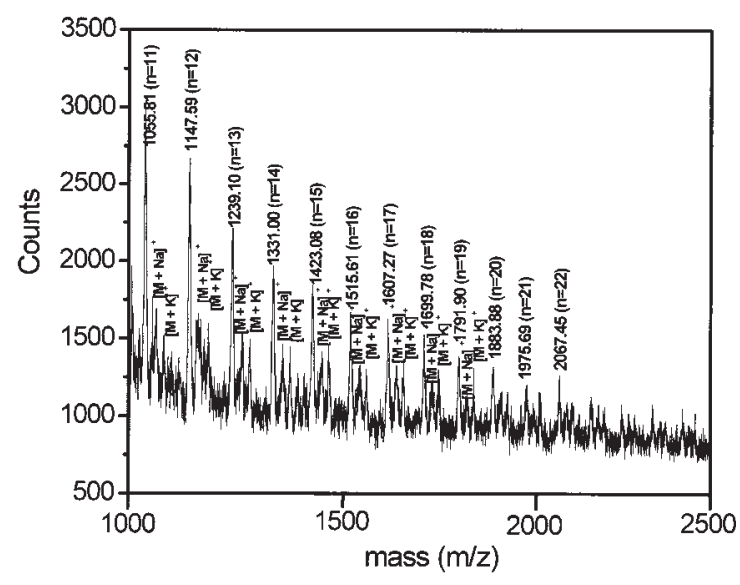

Figure 3. MALDI-TOF MS spectra of polyphenol complex with $\operatorname{PEG}\left(M_{\mathrm{n}}=4 \times 10^{2}\right)$.

ular-weight to phenol, which prevents the efficient polymer production.

The molecular weight of the resulting polyphenol was estimated by SEC with UV detector $(340 \mathrm{~nm})$, in which only the polyphenol was detected. ${ }^{8 \mathrm{~b}}$ The molecular weight of the polyphenol was in the range from 1600 to 3800 . The molecular weight of the polyphenol depended on that of PEG. This may be due to the interaction ability of phenol and/or the resulting polymer to PEG. In addition, the polymer formation from phenol in the present reaction system was confirmed by matrix-assisted laser desorption/ionization-time-of-flight mass spectroscopy (MALDI-TOF MS) (Figure 3). In the case of PEG with molecular weight of $4 \times 10^{2}$ (entry 11 ), the peaks of polyphenol with degree of polymerization up to 22 were detected. The peak separation corresponded to the mass of the phenylene or oxyphenylene repeating unit, $92 \mathrm{~m} / z$.

\section{Properties of Polyphenol Complexes with PEG}

The complex formation between the resulting polyphenol and PEG by hydrogen bonding interaction was confirmed by FT IR (Figure 4). For the polyphenol synthesized in an aqueous methanol without PEG, two peaks centered at $3366 \mathrm{~cm}^{-1}$ and $3524 \mathrm{~cm}^{-1}$ were observed: the former due to the self-associated phenolic $\mathrm{O}-\mathrm{H}$ groups and the latter due to the non-associated free hydroxyl groups. In the case of the polyphenol complex with PEG synthesized in a phosphate buffer in the presence of PEG, a peak at $3366 \mathrm{~cm}^{-1}$ due to self-associated phenolic O-H group was shifted to $3289 \mathrm{~cm}^{-1}$, ascribed to phenolic $\mathrm{O}-\mathrm{H}$ hydrogenbonding with PEG. ${ }^{12}$

The DSC analysis of the present complex verified the miscibility between the polyphenol and PEG. In the DSC chart of the complex (Figure 5), no melting point due to PEG was observed. A single glass transition temperature was seen at $43^{\circ} \mathrm{C}$, lower than that of

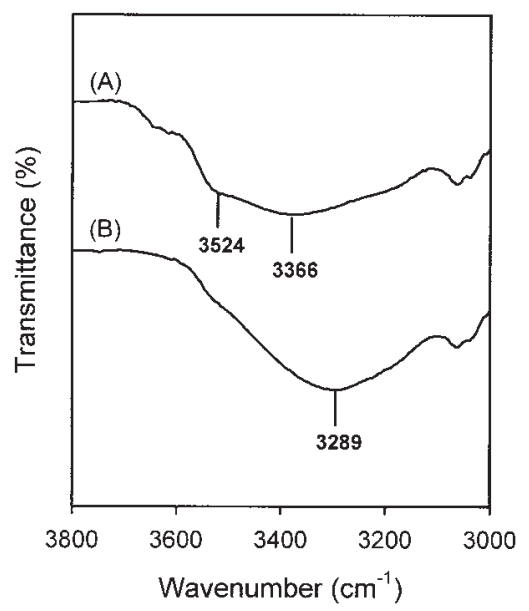

Figure 4. FT IR spectra of (A) a polyphenol synthesized in a mixture of methanol and buffer in the absence of PEG, and (B) polyphenol complex with PEG synthesized in phosphate buffer in the presence of PEG.

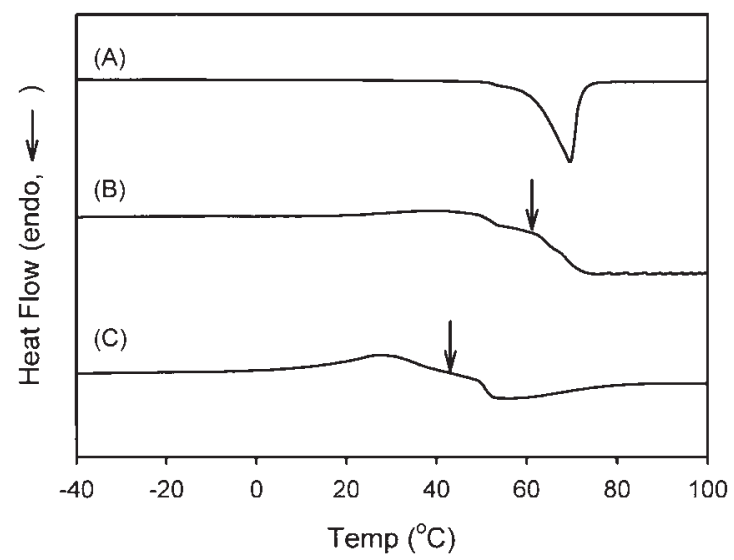

Figure 5. DSC thermograms (second heating) of (A) PEG $\left(M_{\mathrm{n}}=2 \times 10^{4}\right)$, (B) a polyphenol synthesized in a mixture of methanol and buffer in the absence of PEG, and (C) polyphenol complex with PEG synthesized in phosphate buffer in the presence of PEG.

the polyphenol synthesized in an aqueous methanol without PEG $\left(61^{\circ} \mathrm{C}\right) .{ }^{8 \mathrm{~b}}$ These data clearly indicate that there exists rather strong intermolecular interaction between both polymers in the complexes ${ }^{13}$ and they are fully miscible with each other in a homogeneous amorphous phase. Moreover, the glass transition temperature slightly increased as the molecular weight of the PEG template decreased, i.e., the phenylene unit content of the polyphenol increased (Figure 6).

Crystallographic analysis was carried out using Xray diffraction (XRD) for elucidating the change of crystallinity between PEG and the polyphenol complex with PEG (data not shown). The diffraction trace of PEG showed the clear crystalline diffraction peaks at $2 \theta=10-40^{\circ}$. For the complex, on the other hand, the crystalline diffraction peaks of PEG disappeared 


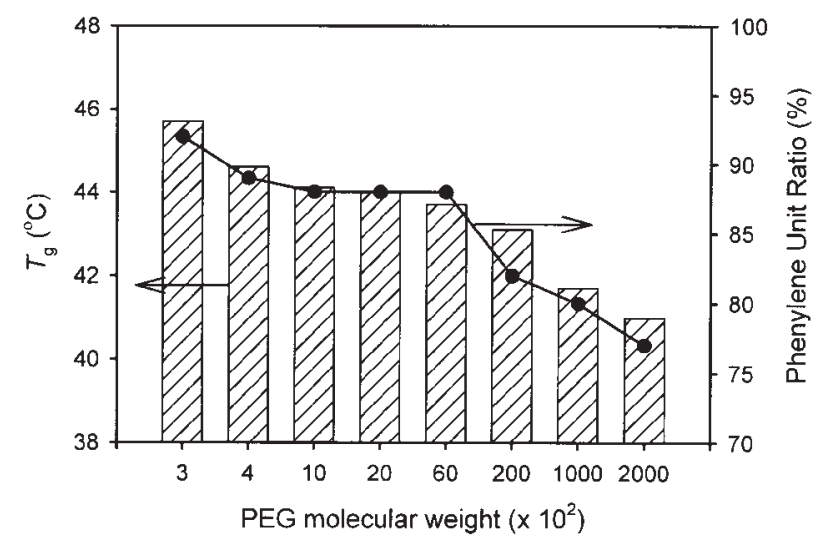

Figure 6. Effect of molecular weight of PEG on glass transition temperature $\left(T_{\mathrm{g}}\right)$ and structure of polymer.

and the broad scattering appeared at $2 \theta=21^{\circ}$. These data clearly support that the polyphenol synthesized in a buffer with PEG was a miscible complex of polyphenol and PEG possessing a homogeneous amorphous phase.

\section{CONCLUSIONS}

The HRP-catalyzed polymerization of phenol efficiently proceeded in water in the presence of some WSPs to form a complex of the product phenolic polymer with WSP. Among them, the polymer yield was the highest when using PEG as template. The addition of PEG enormously improved the regioselectivity to give the polymer mainly consisting of phenylene unit. The hydrogen bonding interaction of the present complex was confirmed by the FT IR, DSC, and XRD analyses. The present study clearly shows that the carbon-carbon coupling between phenolic radical intermediates regioselectively takes place in the presence of PEG, PMOZ or PEOZ in an aqueous medium. Furthermore, an environmentally benign system of the polyphenol production without use of organic solvents has been developed by the addition of these WSPs as template. Further studies on the synthesis of functional polyphenols by the enzymatic template polymerization are under way in our laboratory.

Acknowledgment. This work was partly supported by Program for Promotion of Basic Research Activities for Innovative Bioscience and the 21st Century COE Program, COE for a United Approach to New Materials Science.

\section{REFERENCES}

1. a) Y. Y. Tan and G. Challa, in "Encyclopedia of Polymer Science and Engineering," H. F. Mark, N. M. Bikales, C. G. Overberger, and G. Menges, Ed., Wiley, New York,
N.Y., 1989, vol 16, p 554.

b) S. Połowiński, in "Polymeric Materials Encyclopedia," J. C. Salamone, Ed., CRS Press, Boca Raton, FL, 1996, vol 11, p 8280.

c) S. Połowiński, Prog. Polym. Sci., 27, 537 (2002).

2. a) C. Cristallini, G. Ciardelli, G. Polacco, A. Villani, L. Lazzeri, and P. Giusti, Polym. Int., 48, 1251 (1999).

b) I. Rainaldi, C. Cristallini, G. Ciardelli, and P. Giusti, Polym. Int., 49, 63 (2000).

c) J. Szumilewicz, Macromol. Symp., 161, 183 (2000).

3. For recent reviews on enzymatic synthesis of polyaromatics, see: a) S. Kobayashi, S. Shoda, and H. Uyama, Adv. Polym. Sci., 121, 1 (1995).

b) H. Uyama and S. Kobayashi, CHEMTECH, 29 (10), 22 (1999).

c) S. Kobayashi, J. Polym. Sci., Polym. Chem. Ed., 37, 3041 (1999).

d) R. A. Gross, A. Kumar, and B. Kalra, Chem. Rev., 101, 2097 (2001).

e) S. Kobayashi, H. Uyama, and M. Ohmae, Bull. Chem. Soc. Jpn., 74, 613 (2001).

f) S. Kobayashi, H. Uyama, and S. Kimura, Chem. Rev., 101, 3793 (2001).

4. For recent papers on enzymatic polymerization of phenols, see: a) M. H. Reihmann and H. Ritter, Macromol. Biosci., 1, 85 (2001).

b) H. Uyama, N. Maruichi, H. Tonami, and S. Kobayashi, Biomacromolecules, 3, 187 (2002).

c) L. Mejias, M. H. Reihmann, S. Sepulveda-Boza, and H. Ritter, Macromol. Biosci., 2, 24 (2002).

d) P. Xu, J. Kumar, L. Samuelson, and A. L. Cholli, Biomacromolecules, 3, 889 (2002).

e) T. Fukuoka, Y. Tachibana, H. Tonami, H. Uyama, and S. Kobayashi, Biomacromolecules, 3, 768 (2002).

f) N. Mita, S. Tawaki, H. Uyama, and S. Kobayashi, Macromol. Biosci., 3, 253 (2003).

g) Y. Pang, H. Ritter, and M. Tabatabai, Macromolecules, 36, 7090 (2003).

5. a) T. Oguchi, S. Tawaki, H. Uyama, and S. Kobayashi, Macromol. Rapid Commun., 20, 401 (1999).

b) T. Oguchi, S. Tawaki, H. Uyama, and S. Kobayashi, Bull. Chem. Soc. Jpn., 73, 1389 (2000).

6. a) H. Tonami, H. Uyama, S. Kobayashi, M. Reihmann, and H. Ritter, e-Polymers, no. 3 (2002).

b) N. Mita, S. Tawaki, H. Uyama, and S. Kobayashi, Macromol. Biosci., 2, 127 (2002).

7. F. F. Bruno, R. Nagarajan, P. Stenhouse, K. Yang, J. Kumar, S. K. Tripathy, and L. A. Samuelson, J. Macromol. Sci., Part A: Pure Appl. Chem., 38, 1417 (2001).

8. a) F. F. Bruno, R. Nagarajan, J. Kumar, and L. A. Samuelson, J. Macromol. Sci., Part A: Pure Appl. Chem., 39, 1061 (2002).

b) Y. J. Kim, H. Uyama, and S. Kobayashi, Macromolecules, 36, 5058 (2003).

9. S. Kobayashi, H. Uyama, N. Higuchi, and T. Saegusa, Macromolecules, 23, 54 (1990).

10. N. Mita, S. Tawaki, H. Uyama, and S. Kobayashi, Chem. Lett., 402 (2002).

11. a) H. Shimizu, A. Kaito, and M. Hatano, Bull. Chem. Soc. 
Jpn., 54, 513 (1981).

b) V. K. Smith, T. T. Ndou, and I. M. Warner, J. Phys. Chem., 98, 8627 (1994).

12. a) E. J. Moskala, D. F. Varnell, and M. M. Coleman, Polymer, 26, 228 (1985).

b) J. Hong, S. H. Goh, S. Y. Lee, and K. S. Siow, Polymer, 36, 143 (1995).

c) Z. Zhong and Q. Guo, J. Polym. Sci., Part A: Polym.
Chem., 36, 401 (1998).

d) S. H. Goh, S. Y. Lee, Y. T. Yeo, X. Zhou, and K. L. Tan, Macromol. Rapid Commun., 20, 148 (1999).

13. a) T. K. Kwei, J. Polym. Sci., Polym. Lett. Ed., 22, 307 (1984).

b) X. Zhang and D. H. Solomon, Macromolecules, 27, 4919 (1994). 